\title{
The Communication Development in Children and Their Socio-cultural Awareness
}

\author{
Flora Lamcja (Zeqaj) \\ “A. Xhuvani” University of Elbasan, Elbasan, Albania
}

\begin{abstract}
During the recent years, the assessment for the infants and preschoolers with special needs have undergone substantial changes in the way that how the information is collected and shared by the members of their families and by professionals. Some features of the assessment of the communication skills of infants reflect the following tendencies: emphasis on ecological significance to the practical and assessment ways, permanence of assessment ways, ascending emphasis on active participation of family members and other caregivers on collaborative nature of assessment and the intervention planning process. Along with these changes of the assessment practices in the area of communication reflect on a wider observance of what determines the communication to the young children. Socio-cultural descent effects on many communication aspects by involving the ways when and how a child interacts with adults or strangers, the used dialect and the manners how the child understands or observes the communication process. In this study are included children who had communication problems as well as their parents. All the data is gathered about these children in order to have a proper conclusion about the hypothesis raised in this study. Therefore, what was conducted by the study is that the children had serious problems on communication, on corporation with others, on possession/holding certain emotions etc. The situation was evaluated and it brought its conclusion and appropriate recommendations were given for intervention in order to improve the situation of these children. Hypothesis: The socio cultural awareness effects on the development of the children's communication. Research question: Why does socio cultural awareness and alternative communication effect on the development of the children's communication? Methodology: For the realization of this study, there are used data collected from the parents, teachers about the children. There are also used questionnaires which provide specific data about the topic. In this study, there are 10 children and 20 parents of the corresponding children, who presented adequate data about the progress of their children. Parents were interviewed and they share the information about their children and their relation with them.
\end{abstract}

Keywords: communication, development, culture awarness

\section{Introduction}

\section{Socio-cultural Consciousness}

Moreover, in constrast with the traditional ways of assessments and achievements which make up more than one "unique measure", there is a growing recognition that professionals should be more sensitive towards the social, cultural and linguistic features and to the children's ethnic diversity and their corresponding families. Professionals are interacting with families of different backgrounds that often differ from their own family. One

Flora Lamcja (Zeqaj), professor-part time, Faculty of Medical Technical Sciences, “A. Xhuvani” University of Elbasan; Ph.D. Candidate in European University of Tirana. 
of challenges in consideration of the child's communication skills is the need or the necessity to understand the effects of the culture on communication.

Socio-cultural descent effects on many communication aspects by involving the ways when and how a child interacts with adults or strangers, the used dialect and the manners how the child understands or observes the communication process. For instance, Chang (1987) observed that children from Asian-American families' communication should be with as few words as possible and non-verbal actions are more critical towards the accurancy of the message. The origin of a child also influences on the types of the materials and toys valid, so for this reason it may affect even on the cognitive and literary experiences.

In contrast with standardized language tests, methods and examples of descriptive language, which are described later, it can be a good alternative. If you use a standardized language test or other methods of linguistics, it is important to be familiarized with the dialect, cultural expectations, community behavior and the child's family in order to determine whether a child is having communication difficulties.

In order to have an impartial assessment some of the followings should be done: establishing ourselves in the child's culture by observing other members of the same cultural group; by asking the adults who use the dialect; by using ethnographic observations; by showing sensitivity to the communication style of the family and by using toys or other materials which are recognized culturally (Nelson, 1998; Paul, 2001). Besides this, Westby (1990) provides guidance to use ethnographic investigations in order to gather information from the families for their concerns and priorities.

\section{Priorities of the Family}

As it is suggested above, it is critical that the evaluation activites are based on what the family wants or needs in order to find things useful. In this context, the concerns of the family are used as contexts for evaluation. There are many reasons that family needs to have an evaluation for their children, for instance, several families want to know why the communication skills of their child are not developing and/or should they do in order to help the child and their own family too. Others may want to know if their child's communication is delaying compared with other children.

There are others who may know the difficulties of their child and they simply want to help in determining which preschool program is the best. Professionals make evaluations for several reasons, for example: in order to achieve the family's objectives, to ensure the right to be chosen at one child, to document the progress of the therapy or to require information to facilitate the planning of the intervention. Although some professionals may be restricted to determining some of these requirements for example (the usage of the two standardized tests) so they should do a careful selection and use of variation of the evaluation measures and techniques because these can give flexibility.

\section{Communication Alternatives}

Children who have severe cognitive, neurological, structural and sensitive problems find it difficult to speak and even to write. On the evaluation process, these children alternate the communication manners like: gestures, facial expressions or the communication systems and these are all necessary. Additional terms and the communication alternatives refer to the usage of one or several supplementary ways of the communication skills that each individual owns.

Strategies (AAC) are implemented to the infants who are 4-6 months and to the children who have severe damages, this system may be critical for the child's development. The AAC is a set of integrated components 
which includes symbols, aids, strategies and techniques which may be used individually to enhance communication. Typical skills examined on the AAC system are the cognitive and sensitive functions, the history of natural speaking, language and imitation skills, forecasting and progress of theraphy, the attitudes and the beliefs of caregivers. There are factors which effect on the decision of what kind of system will be used or which interdisciplinary team is necessary.

\section{Communication Partners}

A necessary part of the whole assessment includes the observation of children in corporation with their partners in their dairy activities. As McLean (1990) suggests, the nature and development of communication is transactional and both partners influence with several types of interaction. The interaction literature-children and adults has shown that behaviors that facilitate communication include directing the attention of the child in order to provide possible anwers, positive models, by imitating or expanding a child's actions or words, by accepting the efforts of the child and by adjusting the environment.

However, the best way is to interact with a child or to encourage the practitioners in order to show the caregivers how they should interact with their children. Moreover, the focus between the caregiver and the child, or the teacher with the child, interacts in order to identify the behaviours that can facilitate this corporation and communication and to encourage the refinement of these behaviors. Being attentive about the interaction of the child's features and the caregiver, individual nature of the interaction of the caregiver and the child, practitioners should be careful in determining the conclusions and suggestions of the interventions.

In addition to this, Plapinger and Kretschmer (1991) have reported that several caregivers' behaviors differ from the context and they are an additional factor for the parents' hopes for their role in several activities. In fact, Sorby and Martlew (1991) report a low incidence of comments created during reading books versus playing with toys. Actually, some children may refuse to give answers to their caregivers. Understanding a speech is also an important factor which is stated by Conti-Ramsden (1990) who examine the unpredictable responses of the children to mothers with or without linguistic problems and they found that children could understand more because their mothers use words which are related to the concrete situations.

According to the suggestions of Conti-Ramsden (1993), the efforts to evaluate and modify the behavior of parental conversation may carry the implication that the parents have lost somehow to ensure their children need or they may have contributed somewhat in this problem. Therefore, according to Conti-Ramsden, it is necessary to share with their families their interactive behaviors which are not wrong and they think that the children with special needs mostly need special help in learning the language. In fact, literature is so clear about the types of strategies which can facilitate the children's communication by sharing some of these strategies with the caregivers in order to be more productive. Positive ideas and thoughts for the examination and the influence of the relations child-caregivers are found by McDonald (1989), McCollum and Yates (1994), Warren and Yoder (1998), Wilcox (1992), Wilcox and Shannon (1998).

\section{Methodology}

For the methodology of this scientific study are used quantitative methods.

\section{The Purpose of Research}

This scientific study investigated the the communication development in children and their socio-cultural awareness. 


\section{Sample}

For the realization of this study, there are used data collected from the parents, teachers about the children. There are also used questionnaires which provide specific data about the topic. In this study, there are 10 children and 20 parents of the corresponding children, who presented adequate data about the progress of their children. Parents were interviewed and they share the information about their children and their relation with them.

\section{Results}

The data collected showed the following the situation: The children who were presented as study cases had serious problem in communication.

Table 1

Descriptive Statistics (Frequency)

\begin{tabular}{|c|c|c|c|c|}
\hline Questions & Not at all & A little & Enough & A lot \\
\hline The child speak with plain words & 3 & 6 & 1 & 0 \\
\hline Communicates verbally in any situation & 4 & 3 & 3 & 0 \\
\hline Use indicative gestures in any conversation & 3 & 4 & 2 & 1 \\
\hline Being emotional in some situations & 3 & 3 & 2 & 2 \\
\hline Feels safe in action & 2 & 6 & 1 & 1 \\
\hline Wants to interact with persons nearby & 3 & 3 & 2 & 2 \\
\hline Appears egocentric & 2 & 4 & 3 & 1 \\
\hline Seeks to be independent & 3 & 5 & 1 & 1 \\
\hline Reacts positively by the additional communication options. & 0 & 2 & 2 & 6 \\
\hline Appears aggressive when communicates & 2 & 4 & 4 & 2 \\
\hline
\end{tabular}

The following conclusions are found from the collected results: Children who were taken in the study, 3 of them do not pronounce plain words, 6 of them pronounce a few words, 1 child pronounce some words and 0 child speaks a lot of words. It was observed that they react positively to the usage of alternatives in communication.

\section{Conclusions}

According to the collected data, we can conclude that children who have communication problems pose problems encountered in close relation with other social and cultural issues. Therefore, these children pose problems regarding emphathy, social interaction, self-esteem and they often show lack of emotional support, initiative to express themselves, emotional state etc. According to the interviews, it is also concluded that these children often were found without their parents' emotional support, physical distance because of their jobs, emotional states that sometimes are passed on the child.

There are not used proper alternative communication ways with children who have communication difficulties because they did not have their social circle in order to take the appropriate impacts for the realization of the verbal and non verbal communication. The information provided by the parents show that these children have not done the proper treatments and necessary instructions for the continuation of the work with these children as special cases, for their immediate treatment in order to observe a second situation for their improvement. 


\section{References}

Cheng, L. (2002a). Asian and Pacific-American cultures. In D. E. Battle (Ed.), Communication disorders in multicultural populations (3rd ed., pp. 71-112).

Conti-Ramsden. (1990). Language development and social interaction in blind children. Retrieved from https://books.google.al/books?id=V0mk6ArX6HIC\&pg=PA144\&lpg=PA144\&dq=Conti-Ramsden+(1990)\&source=bl\&ots= Pbii-eCvBB\&sig=jxkmk7RXaE4nFMEBOYxoJfA9Gn8\&hl=en\&sa=X\&ved=0ahUKEwjVt7yo7bDQAhVHLsAKHTH7Cx YQ6AEIMzAD\#v=onepage\&q=Conti-Ramsden\%20(1990)\&f=false

Gutierrez-Clellen, V. F. (1996). Language diversity: Implications for assessment. In K. N. Cole, P. S. Dale, \& D. J. Thal (Eds.), Assessment of communication and language (Vol. 6, pp. 29-56). Baltimore: Paul H. Brookes.

McCollum, J. A., \& Yates, T. J. (1994). Dyad as focus, triad as means: A family centered approach to supporting parent-child interaction. Infants and Young Children, 54-63.

Nelson (1998); Paul (2001). Controversial therapies for developmental disabilities By John W. Jacobson, Richard M. Foxx, James A. (pp. 223). Retrieved from https://books.google.al/books?id=nfZ4AgAAQBAJ\&pg=PA223\&lpg=PA223\&dq=Nelson, +1998;+Paul,+2001\&source=bl\&ots=JiwTD7ne5P\&sig=664OrqNQzNXD2jh9UjiLRuytaWE\&hl=en\&sa=X\&ved=0ahUKE wjuwPTL6rDQAhWrKMAKHcD4A00Q6AEIKzAG\#v=onepage\&q=Nelson\%2C\%201998\%3B\%20Paul\%2C\%202001\&f= false

Plapinger, \& Kretschmer. (1991). You and your deaf child: A self-help guide for parents. Retrieved from https://books.google.al/books?id=KeyzD5Wx77QC\&pg=PA50\&lpg=PA50\&dq=Plapinger+and+Kretschmer+(1991)\&source =bl\&ots=E5mhfVk5yG\&sig=A46s_6-uHtsS9FaBIR8qPQJ6Uoo\&hl=en\&sa=X\&ved=0ahUKEwiE2qiw7LDQAhUMBcAK HaZ3Ch8Q6AEIKDAC\#v=onepage\&q=Plapinger\%20and\%20Kretschmer\%20(1991)\&f=false

Warren, S. F., \& Yoder, P. J. (1998). Facilitating the transition from preintentional to intentional communication. In A. Wetherby, S. Warren, \& J. Reichle (Eds.), Transitions in prelinguistic communication: Preintentional to intentional and presymbolic to symbolic (pp. 365-384). Baltimore: Brookes.

Westby. (1990). Language development: Foundations, processes, and clinical applications (Seton Hall University, South Orange New Jersey).

Wilcox, \& Shannon. (1998). Child language development: Learning to talk. Retrieved from https://books.google.al/books?id=wRSRRMm6sHIC\&pg=PA157\&lpg=PA157\&dq=Wilcox+and+Shannon+(1998).\&source =bl\&ots=dxW7BiIFV6\&sig=I6Sr3gnYTSj1AUtfrzfnxEW6hYs\&hl=en\&sa=X\&ved=0ahUKEwjvxvjk7bDQAhVIPRoKHX2 nB3gQ6AEIHTAB\#v=onepage\&q=Wilcox\%20and\%20Shannon\%20(1998).\&f=false 